\title{
Inhibition of Pseudomonas aeruginosa Biofilm Formation by Traditional Chinese Medicinal Herb Herba patriniae
}

\author{
Bo Fu, ${ }^{1}$ Qiaolian Wu, ${ }^{1}$ Minyan Dang, ${ }^{1}$ Dangdang Bai, ${ }^{1}$ Qiao Guo, \\ Lixin Shen, ${ }^{1}$ and Kangmin Duan ${ }^{1,2}$ \\ ${ }^{1}$ Key Laboratory of Resources Biology and Biotechnology in Western China, Ministry of Education, College of Life Sciences, \\ Northwest University, Xian, Shaanxi 710069, China \\ ${ }^{2}$ Department of Oral Biology \& Medical Microbiology, Rady Faculty of Health Sciences, University of Manitoba, 780 Bannatyne Ave., \\ Winnipeg, MB, Canada R3E OW2
}

Correspondence should be addressed to Lixin Shen; shenlx@nwu.edu.cn and Kangmin Duan; kangmin.duan@umanitoba.ca

Received 16 August 2016; Accepted 5 January 2017; Published 9 March 2017

Academic Editor: P. Nithyanand

Copyright (C) 2017 Bo Fu et al. This is an open access article distributed under the Creative Commons Attribution License, which permits unrestricted use, distribution, and reproduction in any medium, provided the original work is properly cited.

\begin{abstract}
New antimicrobial agents are urgently needed to treat infections caused by drug-resistant pathogens and by pathogens capable of persisting in biofilms. The aim of this study was to identify traditional Chinese herbs that could inhibit biofilm formation of Pseudomonas aeruginosa, an important human pathogen that causes serious and difficult-to-treat infections in humans. A luxCDABE-based reporter system was constructed to monitor the expression of six key biofilm-associated genes in $P$. aeruginosa. The reporters were used to screen a library of 36 herb extracts for inhibitory properties against these genes. The results obtained indicated that the extract of Herba patriniae displayed significant inhibitory effect on almost all of these biofilm-associated genes. Quantitative analysis showed that $H$. patriniae extract was able to significantly reduce the biofilm formation and dramatically altered the structure of the mature biofilms of $P$. aeruginosa. Further studies showed $H$. patriniae extract decreased exopolysaccharide production by P. aeruginosa and promoted its swarming motility, two features disparately associated with biofilm formation. These results provided a potential mechanism for the use of $H$. patriniae to treat bacterial infections by traditional Chinese medicines and revealed a promising candidate for exploration of new drugs against $P$. aeruginosa biofilm-associated infections.
\end{abstract}

\section{Introduction}

Pseudomonas aeruginosa is a remarkably adaptive bacterial pathogen which can cause persistent infections in burn patients, immune-compromised patients, and individuals with the genetic disease cystic fibrosis. It is one of the most prevalent nosocomial pathogens, and the infections caused by $P$. aeruginosa can be very serious and life-threatening [1].

Biofilm formation is a major characteristic of $P$. aeruginosa chronic infections $[2,3]$. P. aeruginosa cells in biofilms are surrounded by exopolysaccharides and form a structured aggregates, and these cells exhibit increased resistance to antibiotics and other adversary agents [3-6]. Infections caused by biofilm-forming $P$. aeruginosa, such as those in cystic fibrosis lung, are almost impossible to eradicate [7]. There is an urgent need to find novel antimicrobial agents to control such infections $[8,9]$.
Traditional Chinese medicines (TCMs) have been widely used to treat infectious diseases for more than a thousand years in China. Many components constituents of TCMs have been found to be very effective in treating bacterial infections such as gastritis, stomatitis, dermatitis, and bacterial pneumonia [10]. However, the mechanisms of these herbs in treatment infectious diseases are mostly unknown.

Herba patriniae is a perennial herbal of TCM, which contains various beneficial ingredients such as amino acids, vitamins, minerals, alkaloids, tannins, and saponins. It has been reported to have functions of antioxidant, antibacterial, antiviral, blood-activating and stasis-eliminating, promoting regeneration of liver cells, and anxiety-alleviating. The boiling water extracts of $H$. patriniae had been identified having anticyanobacteria activity against Microcystis aeruginosa [11].

In this study, 36 extracts of 18 Chinese herbs that are commonly used for treating infection-like symptoms were 
TABLE 1: Bacterial strains used in this study.

\begin{tabular}{|c|c|c|}
\hline Strains/plasmids & Description & Source \\
\hline E. coli $\mathrm{DH} 10 \mathrm{~B}$ & $\begin{array}{l}\text { F-mcrA(mrr-hsdRMS-mcrBC)80dlacZ } \Delta \text { M15 } \Delta \text { lacX74 deoR } \\
\text { recAl endA1 araD139 } \Delta \text { (ara leu)7697 galU galK } \lambda \text {-rpsl nupG }\end{array}$ & Invitrogen \\
\hline P. aeruginosa $\mathrm{PAO} 1$ & Wild type & This lab \\
\hline pMS402 & $\begin{array}{l}\text { Expression reporter plasmid carrying the promoterless } \\
\operatorname{lu} x C D A B E ; \operatorname{Kan}^{\mathrm{r}}, \operatorname{Tmp}^{\mathrm{r}}\end{array}$ & This lab \\
\hline pKD-pslM & pMS402 containing pslM promoter region; $\operatorname{Kan}^{\mathrm{r}}, \mathrm{Tm}^{\mathrm{r}}$ & This study \\
\hline pKD-pelA & pMS402 containing pelA promoter region; $\operatorname{Kan}^{\mathrm{r}}, \mathrm{Tmp}^{\mathrm{r}}$ & This study \\
\hline pKD-algU & pMS402 containing algU promoter region; $\operatorname{Kan}^{\mathrm{r}}, \mathrm{Tm}^{\mathrm{r}}$ & This study \\
\hline $\mathrm{pKD}-p p y R$ & pMS402 containing $p p y R$ promoter region; $\operatorname{Kan}^{\mathrm{r}}, \operatorname{Tm}^{\mathrm{r}}$ & This study \\
\hline pKD-algA & pMS402 containing $\operatorname{alg} A$ promoter region; $\operatorname{Kan}^{\mathrm{r}}, \operatorname{Tmp}^{\mathrm{r}}$ & This study \\
\hline $\mathrm{pKD}-b d l A$ & pMS402 containing bdlA promoter region; $\operatorname{Kan}^{\mathrm{r}}, \mathrm{Tm}^{\mathrm{r}}$ & This study \\
\hline
\end{tabular}

TABLE 2: Reporter genes and primer sequences used.

\begin{tabular}{|c|c|c|c|c|}
\hline $\begin{array}{l}\mathrm{PA} \\
\text { number }\end{array}$ & Gene & Function & Primer & Sequence $\left(5^{\prime} \rightarrow 3^{\prime}\right)$ \\
\hline \multirow{2}{*}{ PA0762 } & \multirow{2}{*}{$\operatorname{alg} U$} & \multirow{2}{*}{ RNA polymerase sigma factor } & $\mathrm{pKD}-\operatorname{alg} U-\mathrm{S}$ & GCACTCGAGAGGATGCCTGAAGACCTC \\
\hline & & & $\mathrm{pKD}-\operatorname{alg} U-\mathrm{A}$ & GTAGGATCCGATGGCGATCCGATACAG \\
\hline \multirow{2}{*}{ PA2243 } & \multirow{2}{*}{ pslM } & \multirow{2}{*}{$\begin{array}{l}\text { Succinate dehydrogenase; } \\
\text { fumarate reductase flavoprotein }\end{array}$} & pKD-pslM-S & ATCCTCGAGCGGTGCGCAAGAAGACC \\
\hline & & & pKD-pslM-A & GTTGGATCCCGTAACGCTCGCCCAGTT \\
\hline \multirow{2}{*}{ PA3064 } & \multirow{2}{*}{ pelA } & \multirow{2}{*}{$\begin{array}{l}\text { Glycoside hydrolase; } \\
\text { deacetylase }\end{array}$} & pKD-pelA-S & CGTCTCGAGCTTTCCACTTTGCCACAG \\
\hline & & & pKD-pelA-A & TACGGATCCTACCAGAACGCCACGCT \\
\hline \multirow{2}{*}{ PA3551 } & \multirow{2}{*}{$\operatorname{alg} A$} & \multirow{4}{*}{$\begin{array}{l}\text { Phosphomannose isomerase; } \\
\text { guanosine } 5^{\prime} \text {-diphospho-D-mannose } \\
\text { pyrophosphorylase } \\
\text { psl and pyoverdine operon regulator }\end{array}$} & $\mathrm{pKD}-\operatorname{alg} A-\mathrm{S}$ & 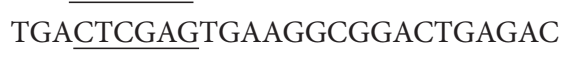 \\
\hline & & & $\mathrm{pKD}-\operatorname{alg} A-\mathrm{A}$ & TATGGATCCGCGACCAGTTTCATC \\
\hline \multirow{2}{*}{ PA2663 } & \multirow{2}{*}{ ppyR } & & $\mathrm{pKD}-p p y R-\mathrm{S}$ & GCACTCGAGCACTTCTTCTGCTACAGC \\
\hline & & & $\mathrm{pKD}-p p y R-\mathrm{A}$ & GTAGGATCCAGCACTTGCACAGCAGAC \\
\hline \multirow{2}{*}{ PA1423 } & \multirow{2}{*}{$b d l A$} & \multirow{2}{*}{ Biofilm dispersion locus A } & $\mathrm{pKD}-b d l A-\mathrm{S}$ & GCACTCGAGCGTCATATTTCCGACGAA \\
\hline & & & $\mathrm{pKD}-b d l A-\mathrm{A}$ & TCAGGATCCGTAGTCTTCCGATTGCG \\
\hline
\end{tabular}

screened for inhibitory effect against $P$. aeruginosa biofilms. We found that the extract of the Chinese herb $H$. patriniae significantly inhibited the expression of the genes associated with biofilm formation in $P$. aeruginosa PAO1. It reduced exopolysaccharide production and biofilm formation and then altered the structure of the mature biofilms.

\section{Materials and Methods}

2.1. Bacterial Strains and Culture Conditions. Bacterial strains used in this study are listed in Table 1 . All the strains were cultured in LB (Luria Bertani) broth at $37^{\circ} \mathrm{C}$ with orbital shaking at $200 \mathrm{rpm}$ or on LB agar plates supplemented with antibiotic of kanamycin $(\mathrm{Kan}, 50 \mu \mathrm{g} / \mathrm{mL})$ or trimethoprim (Tmp, $300 \mu \mathrm{g} / \mathrm{mL}$ ) where appropriate.

2.2. Traditional Chinese Medicine Extraction. Traditional Chinese medicinal herbs were selected according to their efficacy in treatment of infection-like symptoms in Chinese medicine. They were obtained from local pharmacy (Yikang
Pharm chain store, China). The pulverized herb was firstly immerged in $75 \%$ ethanol or deionized water, respectively, for $2 \mathrm{~h}$ and then boiled for $2 \mathrm{~h}$ additionally (weight to solvent volume was 1:5). The extracts were filtered by filter paper and were evaporated under vacuum at $35^{\circ} \mathrm{C}$ using a rotary evaporator (Buchi, Switzerland). The concentrated extracts were then freeze-dried using a lyophilizer and stored at $-80^{\circ} \mathrm{C}$. The water extracts were redissolved in deionized water and ethanol extracts were redissolved in methanol and then sterilized immediately with $0.22 \mu \mathrm{m}$ Iwaki filter before use.

2.3. Gene Expression Assay. Key genes algU [12], pslM [13, 14], pelA [15], algA [16], ppyR [17], and bdlA [18] that are known to be involved in biofilm formation in $P$. aeruginosa were selected to construct luxCDABE-based promoter-reporter fusions (Table 2). The reporters were constructed as previously described $[19,20]$ and were subsequently transformed into PAO1. Using luxCDABE-based reporters, gene expression in liquid cultures was measured by light luminescence (in counts per second) in a Victor ${ }^{3}$ multilabel plate reader 
(Perkin-Elmer). Strains containing different reporters were cultivated overnight in LB broth supplemented with Tmp $(300 \mu \mathrm{g} / \mathrm{mL})$ and then diluted to an optical density at $600 \mathrm{~nm}$ of 0.2. The diluted cultures were used as inoculants. After additional $2 \mathrm{~h}$ incubation, $5 \mu \mathrm{L}$ cultures were inoculated into parallel wells on a 96-well black plate containing $93 \mu \mathrm{L}$ medium and $2 \mu \mathrm{L}$ herbal extract in different concentrations. $50 \mu \mathrm{L}$ mineral oil was added to the wells to prevent evaporation. Promoter activities were measured every $30 \mathrm{~min}$ for $24 \mathrm{~h}$ in a Victor multilabel plate reader and bacterial growth was monitored by measuring $\mathrm{OD}_{600}$ at the same time.

2.4. Gene Expression Measurement by Real-Time Quantitative PCR (RT-qPCR). Bacterial RNA was extracted using RNAprep Pure Cell/Bacteria Kit (TIANGEN). $1 \mu \mathrm{g}$ of RNA sample was reverse transcribed to cDNA using 1st Strand cDNA Synthesis Kit (Takara). Real-time qPCR was performed using SuperReal PerMix Plus (SYBR Green/probe) (TIANGEN) and primers specific for algU (Forward: $5^{\prime}$ AACACCGCGAAGAACCACCT-3', Reverse: 5' -ATCTCATCCCGCAACATCGCG-3'), algA (Forward: $5^{\prime}$-AGAACTGAAGAAGCACGACG-3', Reverse: $5^{\prime}$-TTCTCCATCACCGCGTAGT-3'), pelA (Forward: $5^{\prime}$-ATGGCTGAAGGTATGGCTG-3', Reverse: 5'-AGGTGCTGGAGGACTTCATC-3'), and $p s l M$ (Forward: $5^{\prime}$-CTATGACGCACGGCAACTGG-3', reverse: $5^{\prime}$-CGCCATTGACCAGGTGCAT- $3^{\prime}$ ). The values obtained were normalized to the housekeeping gene proC (Forward: $5^{\prime}$-CAGGCCGGGCAGTTGCTGTC-3 ${ }^{\prime}$, reverse: $5^{\prime}$-GGTCAGGCGCGAGGCTGTCT $-3^{\prime}$ ).

2.5. Quantification of the Biofilm Formation. Biofilm formation was measured in 96-well polystyrene microtiter plate as described previously with minor modifications [21]. $10 \mu \mathrm{L}$ of overnight cultures of PAO1 was added to $90 \mu \mathrm{L}$ fresh LB broth containing $160 \mu \mathrm{g} H$. patriniae water extract. Same volume of deionized water was used as control. After being cultured with shaking at $200 \mathrm{rpm}$ for $1 \mathrm{~h}$ the plates were kept at stationary state at $37^{\circ} \mathrm{C}$ for 3 days and 7 days with a medium replacement at every $24 \mathrm{~h}$ interval. Then wells were washed twice with deionized water gently to remove the planktonic cells. The sedentary cells were stained with $1 \%(\mathrm{v} / \mathrm{v})$ crystal violet solution for $15 \mathrm{~min}$. Unbound dye in the wells were washed off by deionized water before $200 \mu \mathrm{L}$ of $95 \%$ ethanol was added to dissolve the crystal violet stains. The absorbance of the solutions was then measured at $570 \mathrm{~nm}[7,22]$.

2.6. Biofilm Imaging by Silver Staining Method. To examine the biofilm structure, silver staining method was used as described previously with some modifications [23, 24]. Similar to the above described biofilm assay, biofilms were grown on cover slip ( $8 \mathrm{~mm}$ diameter) placed at bottom of the wells of 24 -well plates. $200 \mu \mathrm{L}$ of fresh bacterial culture was inoculated to $1.8 \mathrm{~mL}$ TSB supplemented with $3.2 \mathrm{mg}$ $H$. patriniae. The plates were incubated at $37^{\circ} \mathrm{C}$ with TSB medium replaced every $24 \mathrm{~h}$. After 3 days or 7 days of cultivation, the cover slip was taken out and washed three times with saline water to remove the planktonic cells. The cover slip was immersed in $2.5 \%$ glutaraldehyde for $1 \mathrm{~h}$ for the cells fixation and rinsed with distilled water for $1 \mathrm{~min}$. Then the cover slip was immersed in saturated calcium chloride solution for $15 \mathrm{~min}$ and rinsed with distilled water for $5 \mathrm{~min}$. The biofilms on the cover slip were then stained with $5 \%$ silver nitrate for $15 \mathrm{~min}$, followed by $1 \%$ hydroquinone colourrendering for $2 \mathrm{~min}$ and then rinsed with distilled water for $1 \mathrm{~min}$. Fixation was treated with $5 \%$ sodium thiosulfate for $1 \mathrm{~min}$, followed by a final water rinse. The cover slip was placed on an inverted optical microscope for biofilm structure observation.

2.7. Swarming Motility Assay. Swarming assay was carried out as previously reported [25]. The medium used for swarming motility assay consists of nutrient broth $(0.8 \%)$, glucose $(0.5 \%)$, and agar $(0.5 \%)$. The plates were dried at room temperature overnight before being used. $2 \mu \mathrm{L}$ of $P$. aeruginosa $\mathrm{PAO} 1$ culture $(\mathrm{OD} 600=0.5)$ mixed with $106.67 \mu \mathrm{g}$ of $H$. patriniae water extract was spotted onto the swarming plate and the one with deionized water was used as blank control. Plates were incubated at $37^{\circ} \mathrm{C}$ for $24 \mathrm{~h}$ before the swarming diameter was measured.

2.8. Measurement of Exopolysaccharide Production. Overnight culture of PAO1 (OD600 $=0.005$ ) was spotted onto Congo red plates (1\% Tryptone, $1 \%$ agar, $4 \%$ Congo red, and $2 \%$ Coomassie blue) with or without $H$. patriniae water extract. The amount of $H$. patriniae water extract was $64 \mu \mathrm{g}$. The colony morphology and staining were observed after 3 days of incubation at $37^{\circ} \mathrm{C}[22]$.

\section{Results and Discussion}

3.1. Screening for Herbs with Inhibitory Effect on P. aeruginosa Biofilm-Associated Genes. Eighteen traditional Chinese medicinal herbs were selected because of their common usage for infection-like symptoms. Both boiling water extracts and ethanol extracts were obtained and used to screen for antibiofilm activities. Since $P$. aeruginosa biofilm formation is directly associated with the activity of several known genes, we constructed luxCDABE-based reporters to examine the effect of herb extracts on these genes. The effects of the crude extracts on the expression of these biofilm-associated genes ( $\operatorname{alg} U, p s l M$, pelA, algA, $p p y R$, and $b d l A)$ are presented in Table 3. The results indicate that different herbal extracts exhibited various degrees of inhibitory effects on these genes. The water extract of $H$. patriniae showed the most significant effect on the expression of $\operatorname{alg} U, \operatorname{alg} A, p s l M$, and $b d l A$. Examples of the gene expression profiles in the presence of H. patriniae are shown in Figure 1.

To confirm the results obtained from the lux-based reporter assay, real-time qPCR was carried out using bacterial RNA samples isolated in the presence and absence of $H$. patriniae. The results are shown in Table 4 . In agreement with the results from the reporter assay, the mRNA levels of alg $U$, $\operatorname{alg} A, p s l M$, and $b d l A$ in PAO1 were all significantly decreased in the presence of $H$. patriniae extract (at $1.6 \mathrm{mg} / \mathrm{mL}$ ) compared with those in the absence of $H$. patriniae extract. It is noted that the inhibition of $\operatorname{alg} U$ and $\operatorname{alg} A$ was more pronounced in the qPCR assay. 


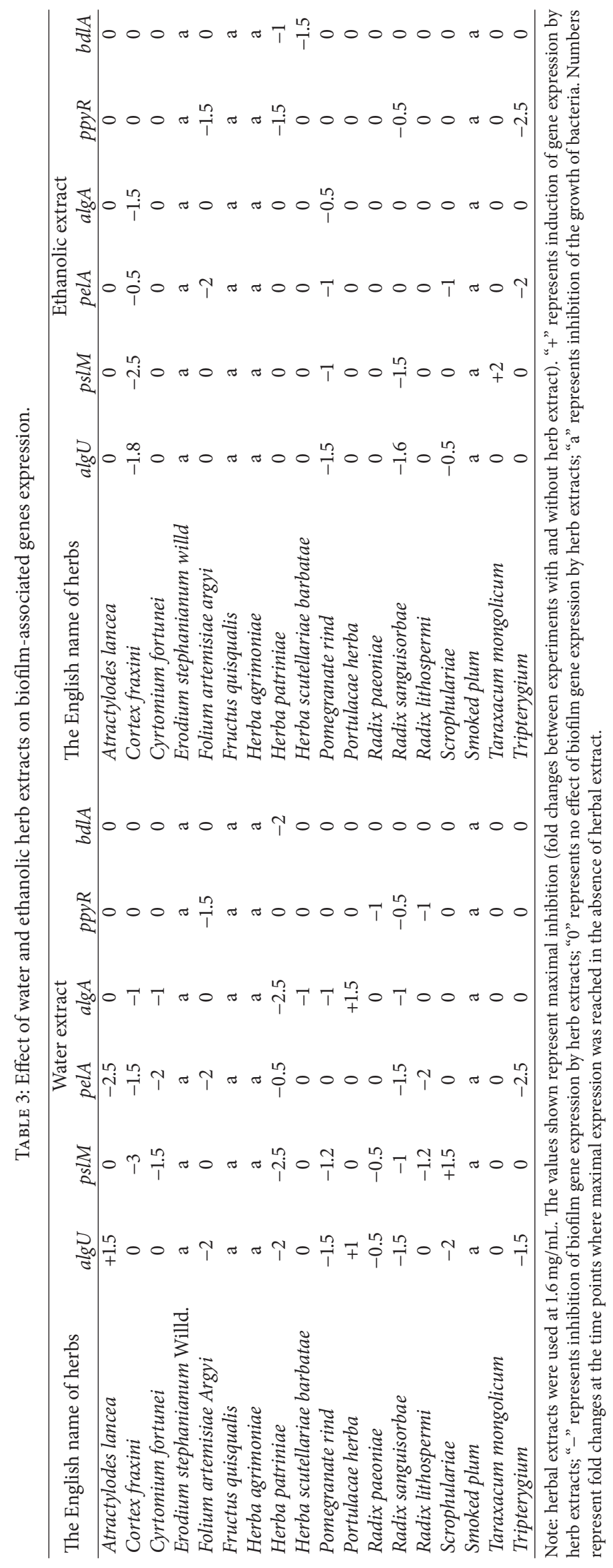



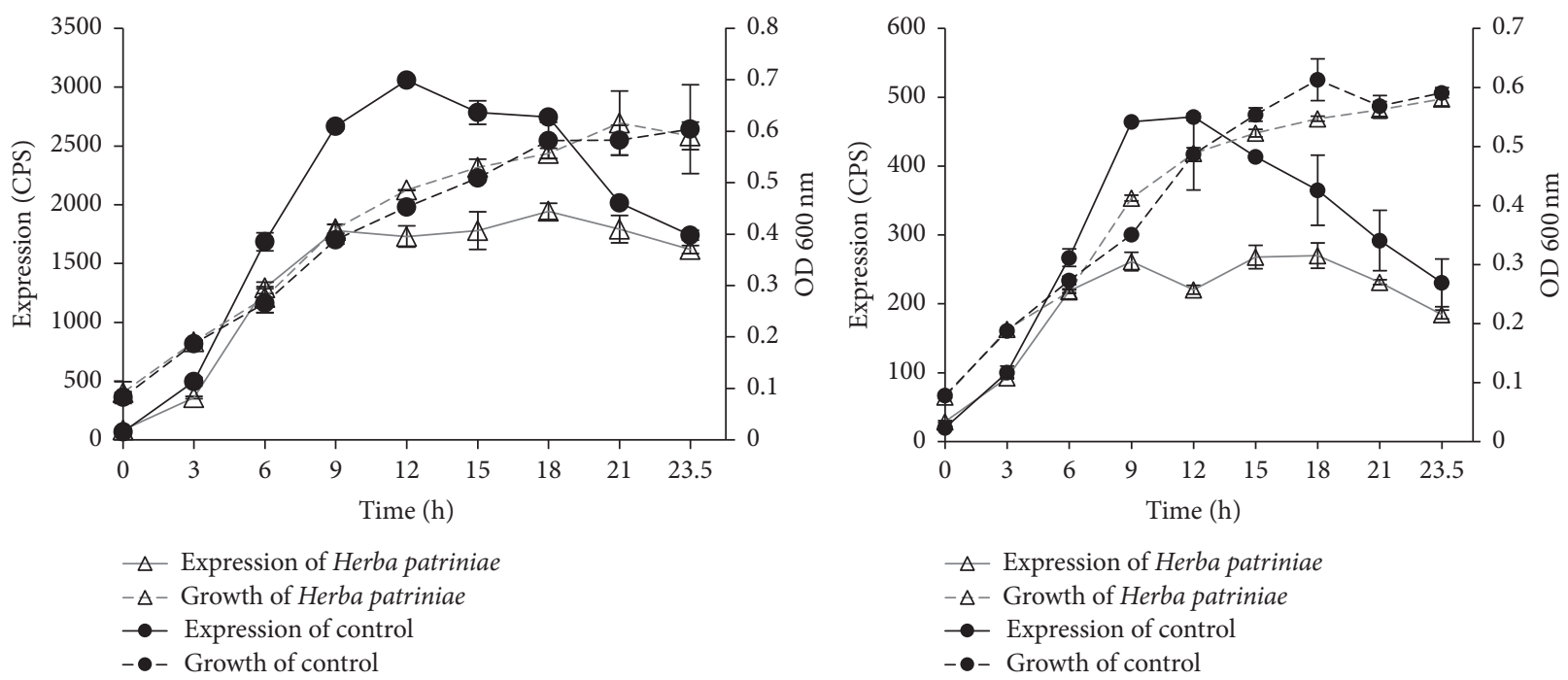

(a)

(b)
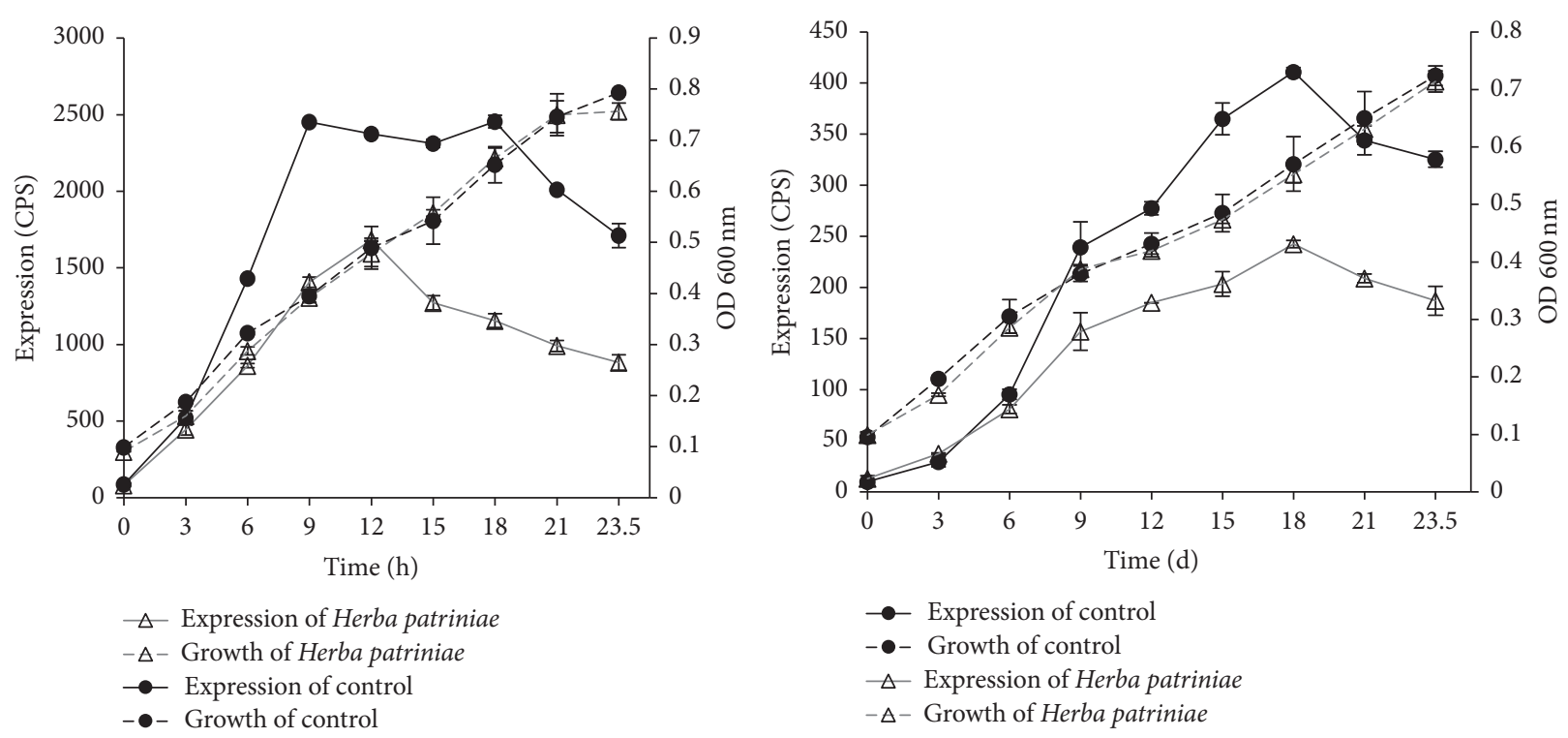

(c)

(d)

FIGURE 1: Expressions of $\operatorname{alg} U$, algA, pslM, and $b d l A$ in medium with water extract of Herba patriniae. Expressions of algU (a), algA (b), pslM (c), and bdlA (d) in medium (total volume of $100 \mu \mathrm{L}$ ) with $160 \mu \mathrm{g}$ H. patriniae extract and the controls were the ones without herbal extract.

The results indicate that many of these herbs have an inhibitory effect on the genes associated with biofilm formation in $P$. aeruginosa. This is somewhat not surprising because they all have been used for treatment of chronic bacterial infections in traditional Chinese medicine.

The effect from the water extract of $H$. patriniae was remarkable as it inhibited five genes tested. It has been reported that the herb had antibacterial and antiviral activity. However, it was noted that the water extract did not inhibit the growth of $P$. aeruginosa (Figure 1). Even though conventional antibiotic compounds may exist in $H$. patriniae against other bacteria, this result indicates no such component was present against $P$. aeruginosa, at least not at the concentrations used in our experiments.

Lacking of bacterial killing or growth inhibition activity, however, may not be a weakness of such herbs in treating infectious diseases. As discussed in previous reports [26-29], a promising new class of antipathogenic drugs that target virulence factors and/or biofilm formation instead of killing the pathogens has many advantages in clinical use. First, these antipathogenic drugs theoretically are less likely to render drug resistance in the pathogens because they do not assert 
TABLE 4: The transcriptional levels of selected genes in PAO1 and PAO1 treated with $H$. patriniae.

\begin{tabular}{|c|c|c|c|}
\hline \multirow{2}{*}{ Gene } & \multicolumn{3}{|c|}{ Relative mRNA level determined by $\Delta \mathrm{Ct}$ calculation } \\
\hline & Without $H$. patriniae & With $H$. patriniae & $P$ value \\
\hline $\operatorname{alg} U$ & 1.00 & 0.192 & 0.014 \\
\hline $\operatorname{alg} A$ & 1.00 & 0.090 & 0.011 \\
\hline pslM & 1.00 & 0.695 & 0.043 \\
\hline$b d l A$ & 1.00 & 0.412 & 0.036 \\
\hline
\end{tabular}

Note: the mRNA level of each gene was normalized to that of proC. The values shown represent the mean of three different tests.

a selective pressure on the pathogen's survival. Second, such therapeutics would unlikely affect other nonpathogenic or beneficial bacteria, that is, the microbiome in the host.

3.2. Extract of $H$. patriniae Inhibits P. aeruginosa Biofilm Formation. From the gene expression results, the extract of $H$. patriniae presumably would inhibit the biofilm formation of $P$. aeruginosa. To verify such an effect, $P$. aeruginosa biofilm formation was compared in the presence and absence of $H$. patriniae extract. As shown in Figure 2(a), significantly less biofilm was formed in the presence of the herb extract than that without the extract at the irreversible attachment stage ( $1 \mathrm{~d}$ ) and mature stage ( $3 \mathrm{~d}$ and $7 \mathrm{~d}$ ) after inoculation. The result is in agreement with the gene expression data, suggesting the $H$. patriniae extract could reduce biofilm formation through inhibiting the genes associated with biofilm formation.

Importantly, the addition of the $H$. patriniae also dramatically altered the structure of the biofilms (Figure 2(b)). It appears that the herb extract prevented the formation of mature biofilms, only allowing $P$. aeruginosa to form smaller cell clusters. These results indicate that the water extract of $H$. patriniae indeed was able to inhibit $P$. aeruginosa biofilm formation.

3.3. Water Extract of $H$. patriniae Inhibited P. aeruginosa Exopolysaccharide Production. The exopolysaccharide (EPS) matrix is an important component of biofilm structure [30]. We compared the exopolysaccharide production in the presence of $H$. patriniae extract to those without $H$. patriniae extract. As shown in Figure 3, P. aeruginosa PAO1 cells produced more EPS shown in red by Congo red staining than the cells with $H$. patriniae extract. This result indicates that $H$. patriniae inhibited $P$. aeruginosa exopolysaccharide production and hence affected biofilm formation.

3.4. H. patriniae Water Extract Promoted PAO1 Swarming Motility. Upon encountering a surface, the surfaceassociated behaviors of $P$. aeruginosa, such as biofilm formation and swarming, are often coregulated [31]. In P. aeruginosa, swarming motility is reversely correlated with biofilm formation. Examination of the swarming motility of PAO1 in the presence and absence of the water extract of $H$. patriniae showed that $H$. patriniae promoted $P$. aeruginosa swarming motility (Figure 4(a)). The diameter of PAO1 grown with $106.67 \mu \mathrm{g} H$. patriniae was almost $5.60 \mathrm{~cm}$, while the control was less than $2.0 \mathrm{~cm}$ (Figure 4(b)). Considering the reverse relationship between swarming motility and biofilm formation, the enhanced swarming motility by $H$. patriniae extract could have contributed the inhibition of biofilm formation.

Taken together, the extract of $H$. patriniae clearly inhibited the biofilm formation of $P$. aeruginosa. It inhibited several key genes $\operatorname{alg} U, p s l M, p e l A, \operatorname{alg} A$, and $b d l A$ that are involved in biofilm formation. $H$. patriniae reduced exopolysaccharide production and promoted swarming motility. Increased motility may reduce adhesion and enable bacteria to actively escape the biofilm matrix to become planktonic bacteria $[13,31,32]$. As depicted in Figure 5, multiple factors/pathways probably have contributed to the reduction of biofilm formation and the altered biofilm structure in the presence of $H$. patriniae.

In a time of resistance to multiple antimicrobial agents in pathogenic bacteria being spread, there is an urgent need to develop new antibacterial agents [1,32]. Drugs against infections that involve biofilms are particularly required. Pathogens in biofilm formation are more resistant to conventional antibiotics and other adversary conditions such as nutritional stress. Biofilms also protect bacterial cells from the activity of host immune response [33, 34].

Traditional Chinese medicines are a valuable source for novel antibacterial agents [35-37]. The inhibitory effect of the water extract of $H$. patriniae against $P$. aeruginosa biofilms and biofilm-related phenotypes signifies that $H$. patriniae is a promising candidate for treatment of infections caused by $P$. aeruginosa biofilms. It could be used in the way of traditional Chinese medicine or it can be explored for active compounds.

\section{Conclusions}

Our results indicate $H$. patriniae extract could significantly inhibit the expression of $P$. aeruginosa genes associated with biofilm formation, alter the structure, and prevent the formation of mature biofilms. It also decreased exopolysaccharide production and promoted swarming motility. These results provided a potential underlying mechanism for the use of $H$. patriniae to treat bacterial infections in traditional Chinese medicine and revealed a promising candidate for exploration of new drugs against $P$. aeruginosa biofilm-associated infections. 


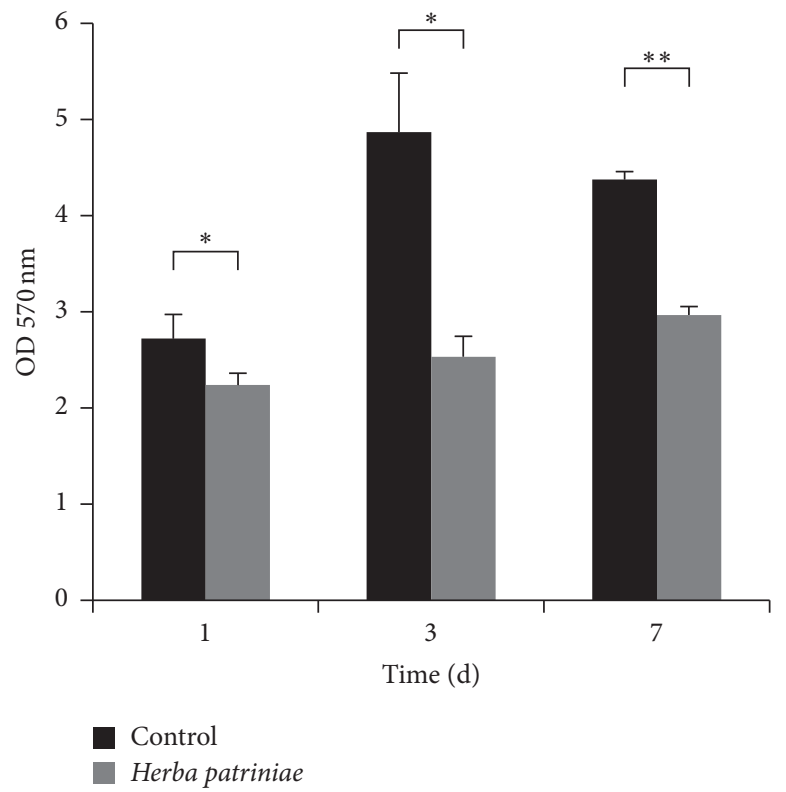

(a)
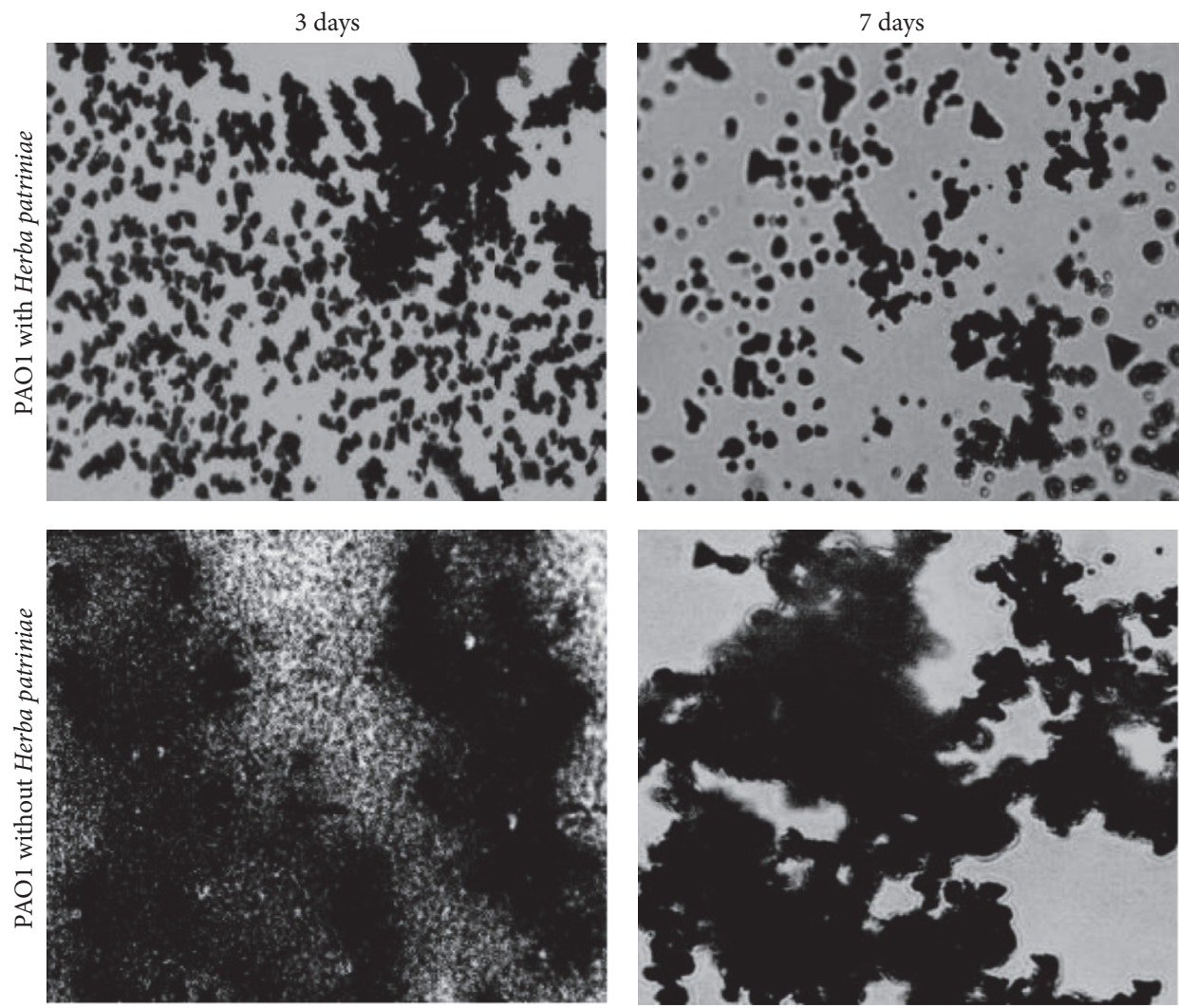

(b)

Figure 2: (a) Inhibition of biofilm production of PAO1 by $H$. patriniae extract. The control did not contain any herb extract. “*” indicates significant difference between the herb group and the control group $(P<0.05)$. “**” indicates very significant difference $(P<0.01)$. (b) Micrographs of biofilms formed with and without $H$. patriniae. Top row, PAO1 silver stained biofilms in the presence of extract of $H$. patriniae after 3 days and 7 days of incubation. Bottom row, PAO1 silver stained biofilms without H. patriniae. 


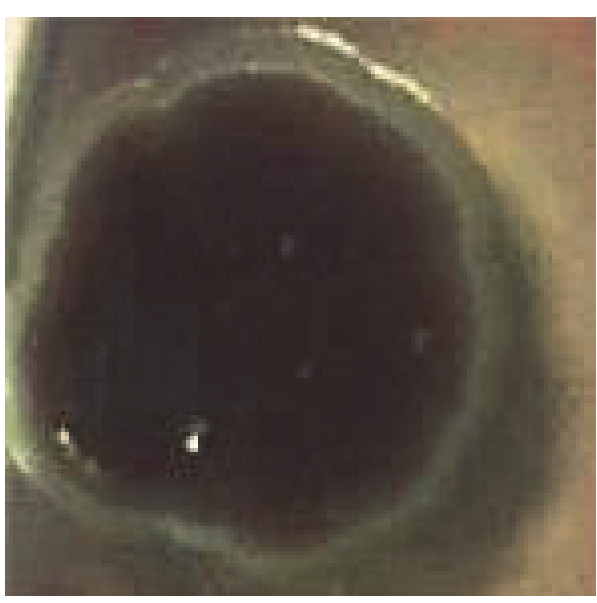

(a)

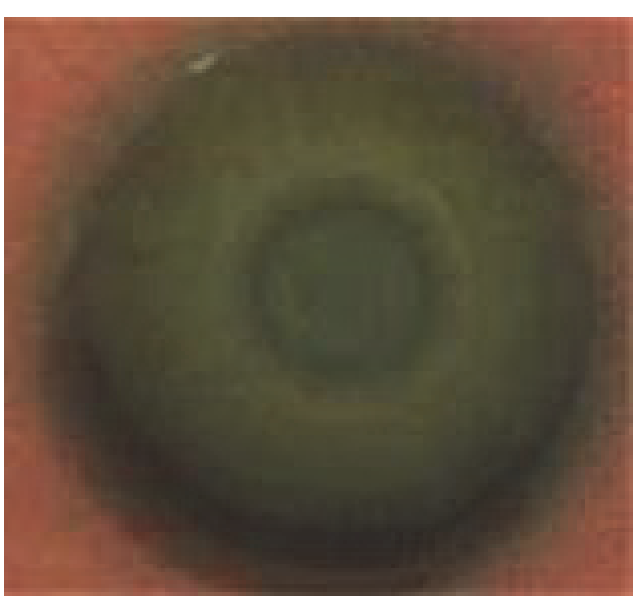

(b)

FIGURE 3: Photograph of exopolysaccharide production on Congo red plates. (a) Without H. patriniae. (b) With H. patriniae. The colony morphology and staining were observed after 3 days of incubation at $37^{\circ} \mathrm{C}$.

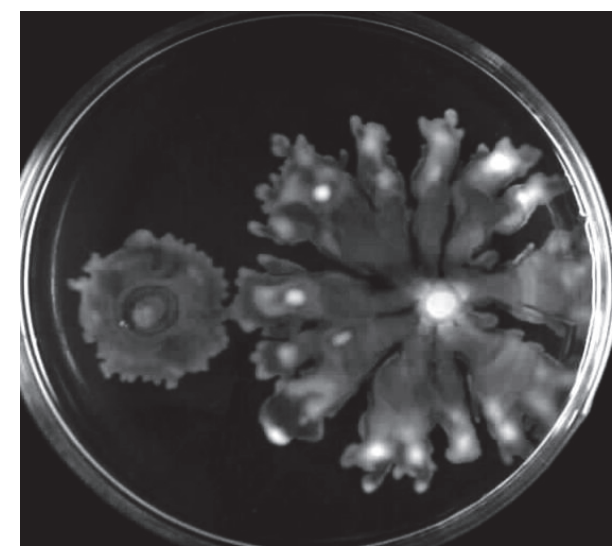

(a)

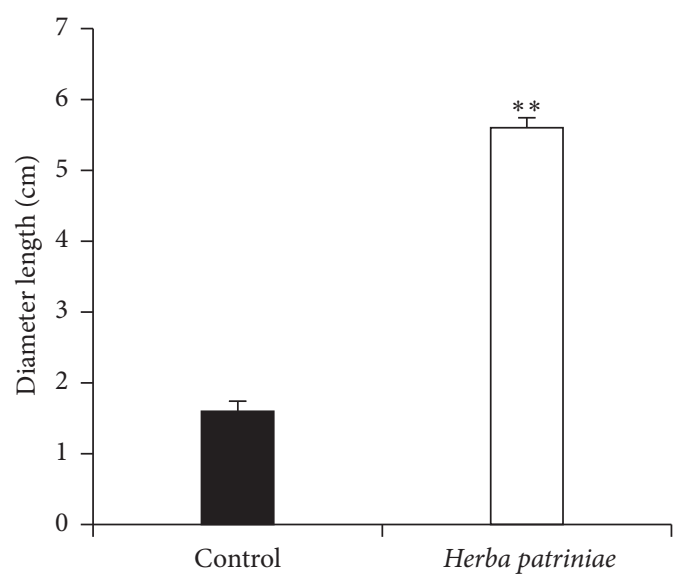

(b)

FIgure 4: Swarming motility of PAO1. (a) PAO1 grown with $H$. patriniae was at right; the one without herb was at left. (b) The swarming diameter of PAO1 with $H$. patriniae, the control was the one without herb. “**” indicates significant difference $(P<0.01)$.

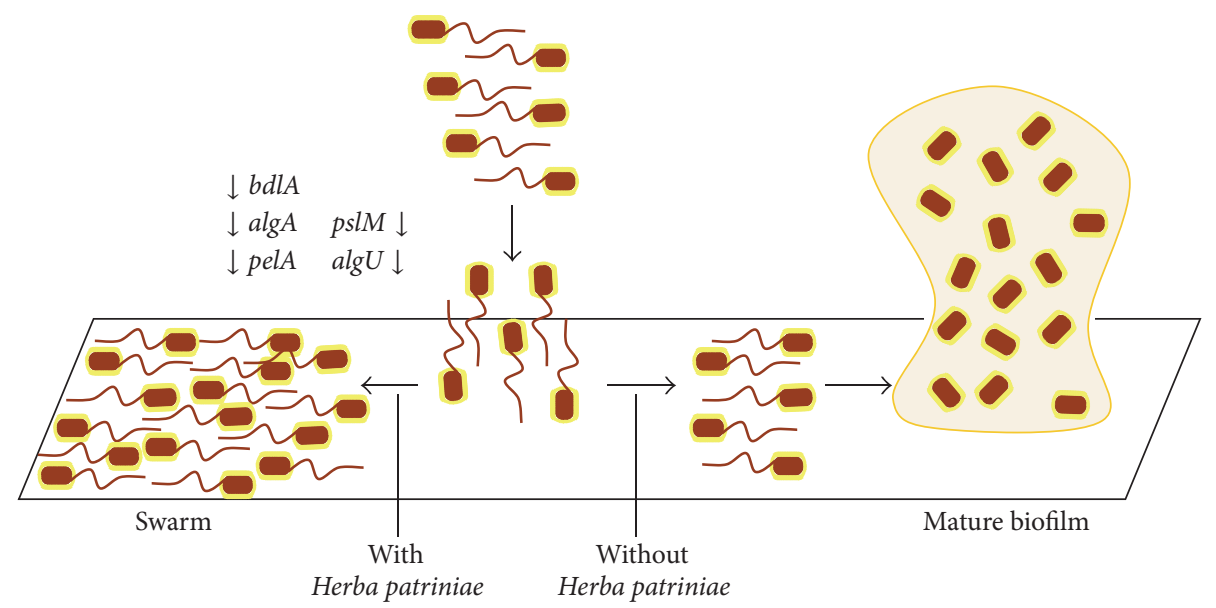

Figure 5: P. aeruginosa biofilm formation and swarming motility in the influence of $H$. patriniae. 


\section{Competing Interests}

The authors declare that they have no competing interests regarding the publication of this paper.

\section{Acknowledgments}

This work was supported by grants from the NSFC (31570131), NSERC (402943-2011 RGPIN), CPSF (2014M562444), and PCSIRT (IRT 15R55).

\section{References}

[1] H. M. Zowawi, P. N. A. Harris, M. J. Roberts et al., "The emerging threat of multidrug-resistant Gram-negative bacteria in urology," Nature Reviews Urology, vol. 12, no. 10, pp. 570-584, 2015.

[2] T. Bjarnsholt, "The role of bacterial biofilms in chronic infections," APMIS. Supplementum, no. 136, pp. 1-51, 2013.

[3] M. Rybtke, L. D. Hultqvist, M. Givskov, and T. TolkerNielsen, "Pseudomonas aeruginosa biofilm infections: community structure, antimicrobial tolerance and immune response," Journal of Molecular Biology, vol. 427, no. 23, pp. 3628-3645, 2015.

[4] N. E. Head and H. Yu, "Cross-sectional analysis of clinical and environmental isolates of Pseudomonas aeruginosa: biofilm formation, virulence, and genome diversity," Infection and Immunity, vol. 72, no. 1, pp. 133-144, 2004.

[5] T.-F. Mah, B. Pitts, B. Pellock, G. C. Walker, P. S. Stewart, and G. A. O'Toole, "A genetic basis for Pseudomonas aeruginosa biofilm antibiotic resistance," Nature, vol. 426, no. 6964, pp. 306-310, 2003.

[6] M. Whiteley, M. G. Bangera, R. E. Bumgarner et al., "Gene expression in Pseudomonas aeruginosa biofilms," Nature, vol. 413, no. 6858, pp. 860-864, 2001.

[7] Q. Guo, W. Kong, S. Jin, L. Chen, Y. Xu, and K. Duan, "PqsRdependent and PqsR-independent regulation of motility and biofilm formation by PQS in Pseudomonas aeruginosa PAO1," Journal of Basic Microbiology, vol. 54, no. 7, pp. 633-643, 2014.

[8] T. Rasamiravaka, Q. Labtani, P. Duez, and M. El Jaziri, “The formation of biofilms by pseudomonas aeruginosa: a review of the natural and synthetic compounds interfering with control mechanisms," BioMed Research International, vol. 2015, Article ID 759348, 17 pages, 2015.

[9] G. H. Talbot, J. Bradley, J. E. Edwards Jr., D. Gilbert, M. Scheid, and J. G. Bartlett, "Bad bugs need drugs: an update on the development pipeline from the antimicrobial availability task force of the infectious diseases society of America," Clinical Infectious Diseases, vol. 42, no. 5, pp. 657-668, 2006.

[10] Q. Guo, S. Jin, J. Xiao, D. Zhang, L. Shen, and K. Duan, "Inhibition of quorum sensing-associated virulence factors in Pseudomonas aeruginosa PAO1 by Folium artemisiae argyi extract," Journal of Pure and Applied Microbiology, vol. 7, no. 1, pp. 261-270, 2013.

[11] J.-D. Yang, L.-B. Hu, W. Zhou, Y.-F. Yin, J. Chen, and Z.-Q. Shi, "Lysis of Microcystis aeruginosa with extracts from chinese medicinal herbs," International Journal of Molecular Sciences, vol. 10, no. 9, pp. 4157-4167, 2009.

[12] A. Bazire, K. Shioya, E. Soum-Soutéra et al., "The sigma factor $\mathrm{AlgU}$ plays a key role in formation of robust biofilms by nonmucoid Pseudomonas aeruginosa," Journal of Bacteriology, vol. 192, no. 12, pp. 3001-3010, 2010.

[13] K. D. Jackson, M. Starkey, S. Kremer, M. R. Parsek, and D. J. Wozniak, "Identification of psl, a locus encoding a potential exopolysaccharide that is essential for Pseudomonas aeruginosa PAO1 biofilm formation," Journal of Bacteriology, vol. 186, no. 14, pp. 4466-4475, 2004.

[14] L. Ma, M. Conover, H. Lu, M. R. Parsek, K. Bayles, and D. J. Wozniak, "Assembly and development of the Pseudomonas aeruginosa biofilm matrix," PLoS Pathogens, vol. 5, no. 3, 2009.

[15] L. Friedman and R. Kolter, "Genes involved in matrix formation in Pseudomonas aeruginosa PA14 biofilms," Molecular Microbiology, vol. 51, no. 3, pp. 675-690, 2004.

[16] U. Remminghorst and B. H. A. Rehm, "In vitro alginate polymerization and the functional role of Alg8 in alginate production by Pseudomonas aeruginosa," Applied and Environmental Microbiology, vol. 72, no. 1, pp. 298-305, 2006.

[17] C. Attila, A. Ueda, and T. K. Wood, "PA2663 (PpyR) increases biofilm formation in Pseudomonas aeruginosa PAO1 through the psl operon and stimulates virulence and quorum-sensing phenotypes," Applied Microbiology and Biotechnology, vol. 78, no. 2, pp. 293-307, 2008.

[18] R. Morgan, S. Kohn, S.-H. Hwang, D. J. Hassett, and K. Sauer, "BdlA, a chemotaxis regulator essential for biofilm dispersion in Pseudomonas aeruginosa," Journal of Bacteriology, vol. 188, no. 21, pp. 7335-7343, 2006.

[19] K. Duan, C. Dammel, J. Stein, H. Rabin, and M. G. Surette, "Modulation of Pseudomonas aeruginosa gene expression by host microflora through interspecies communication," Molecular Microbiology, vol. 50, no. 5, pp. 1477-1491, 2003.

[20] H. Liang, N. Li, Z. Dong, M. G. Surette, and K. Duan, “The YebC family protein PA0964 negatively regulates the Pseudomonas aeruginosa quinolone signal system and pyocyanin production," Journal of Bacteriology, vol. 190, no. 18, pp. 6217-6227, 2008.

[21] G. A. O'Toole and R. Kolter, "Initiation of biofilm formation in Pseudomonas fluorescens WCS365 proceeds via multiple, convergent signalling pathways: a genetic analysis," Molecular Microbiology, vol. 28, no. 3, pp. 449-461, 1998.

[22] W. Kong, L. Chen, J. Zhao et al., "Hybrid sensor kinase PA1611 in Pseudomonas aeruginosa regulates transitions between acute and chronic infection through direct interaction with RetS," Molecular Microbiology, vol. 88, no. 4, pp. 784-797, 2013.

[23] C. Passariello, F. Berlutti, L. Selan, M. C. Thaller, and R. Pezzi, "A rapid staining procedure to demonstrate glycocalyx production and bacterial biofilms," New Microbiology, vol. 17, no. 3, pp. 225230, 1994.

[24] J. Zhao, H. Jiang, W. Cheng et al., "The role of quorum sensing system in antimicrobial induced $\operatorname{ampC}$ expression in Pseudomonas aeruginosa biofilm," Journal of Basic Microbiology, vol. 55, no. 5, pp. 671-678, 2015.

[25] H. Liang, J. Duan, C. D. Sibley, M. G. Surette, and K. Duan, "Identification of mutants with altered phenazine production in Pseudomonas aeruginosa," Journal of Medical Microbiology, vol. 60, no. 1, pp. 22-34, 2011.

[26] S. Yang, Q. Guo, and K. Duan, "Perspectives on antibacterial activities of traditional Chinese medicine and its potential mechanisms," Chinese Journal of Biochemical and Pharmaceutics, vol. 36, no. 4, pp. 15-20, 2016.

[27] M. Otto, "Quorum-sensing control in Staphylococci-a target for antimicrobial drug therapy?" FEMS Microbiology Letters, vol. 241, no. 2, pp. 135-141, 2004. 
[28] A. R. Hauser, J. Mecsas, and D. T. Moir, "Beyond antibiotics: new therapeutic approaches for bacterial infections," Clinical Infectious Diseases, vol. 63, no. 1, pp. 89-95, 2016.

[29] S. Wagner, R. Sommer, S. Hinsberger et al., "Novel strategies for the treatment of Pseudomonas aeruginosa infections," Journal of Medicinal Chemistry, vol. 59, no. 13, pp. 5929-5969, 2016.

[30] D. G. Davies, M. R. Parsek, J. P. Pearson, B. H. Iglewski, J. W. Costerton, and E. P. Greenberg, "The involvement of cell-to-cell signals in the development of a bacterial biofilm," Science, vol. 280, no. 5361, pp. 295-298, 1998.

[31] N. C. Caiazza, J. H. Merritt, K. M. Brothers, and G. A. O’Toole, "Inverse regulation of biofilm formation and swarming motility by Pseudomonas aeruginosa PA14," Journal of Bacteriology, vol. 189, no. 9, pp. 3603-3612, 2007.

[32] Y.-M. Zhou, L. Shao, J.-A. Li et al., "An efficient and novel screening model for assessing the bioactivity of extracts against multidrug-resistant pseudomonas aeruginosa using caenorhabditis elegans," Bioscience, Biotechnology and Biochemistry, vol. 75, no. 9, pp. 1746-1751, 2011.

[33] S. A. Ochoa, A. Cruz-Córdova, G. E. Rodea et al., "Phenotypic characterization of multidrug-resistant Pseudomonas aeruginosa strains isolated from pediatric patients associated to biofilm formation," Microbiological Research, vol. 172, pp. 68-78, 2015.

[34] M. Bala, S. Gupte, P. Aggarwal, M. Kaur, and A. Manhas, "Biofilm producing multidrug resistant Acinetobacter species from a tertiary care hospital: a therapeutic challenge," International Journal of Research in Medical Sciences, vol. 4, no. 7, pp. 3024-3026, 2016.

[35] K. Anani, Y. Adjrah, Y. Ameyapoh et al., "Effects of hydroethanolic extracts of Balanites aegyptiaca (L.) Delile (Balanitaceae) on some resistant pathogens bacteria isolated from wounds," Journal of Ethnopharmacology, vol. 164, pp. 16-21, 2015.

[36] B. P. Marasini, P. Baral, P. Aryal et al., "Evaluation of antibacterial activity of some traditionally used medicinal plants against human pathogenic bacteria," BioMed Research International, vol. 2015, Article ID 265425, 6 pages, 2015.

[37] G. Y. Zuo, G. C. Wang, Y. B. Zhao et al., "Screening of Chinese medicinal plants for inhibition against clinical isolates of methicillin-resistant Staphylococcus aureus (MRSA)," Journal of Ethnopharmacology, vol. 120, no. 2, pp. 287-290, 2008. 

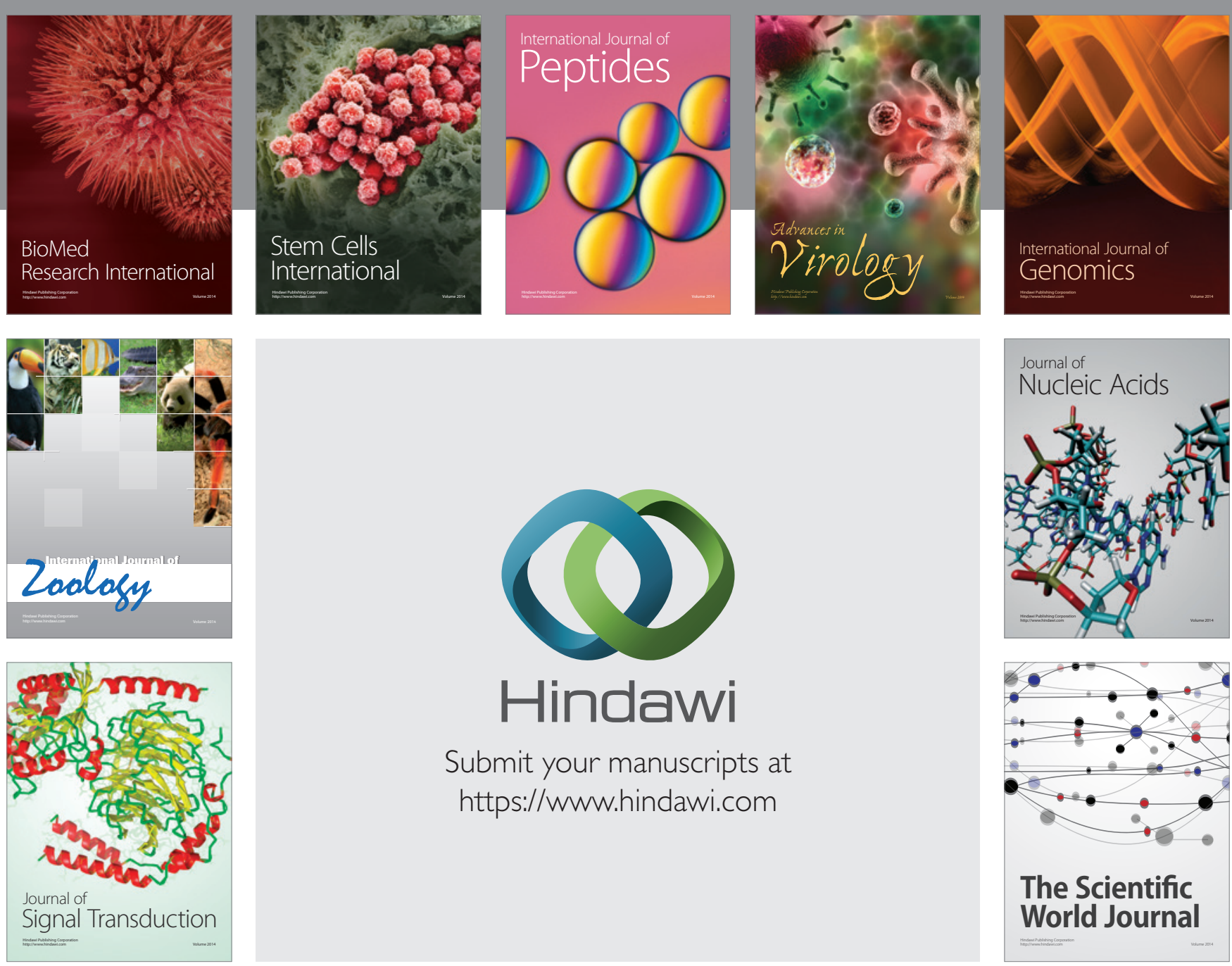

Submit your manuscripts at

https://www.hindawi.com
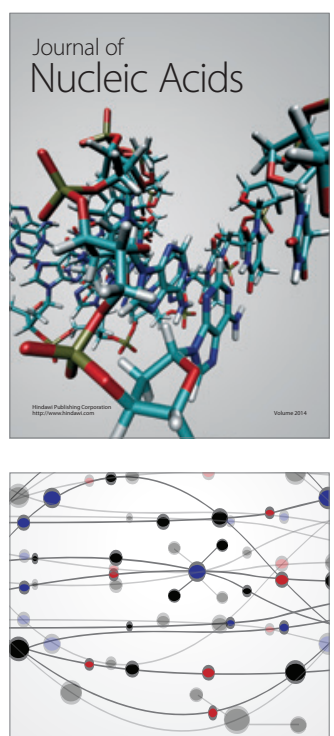

The Scientific World Journal
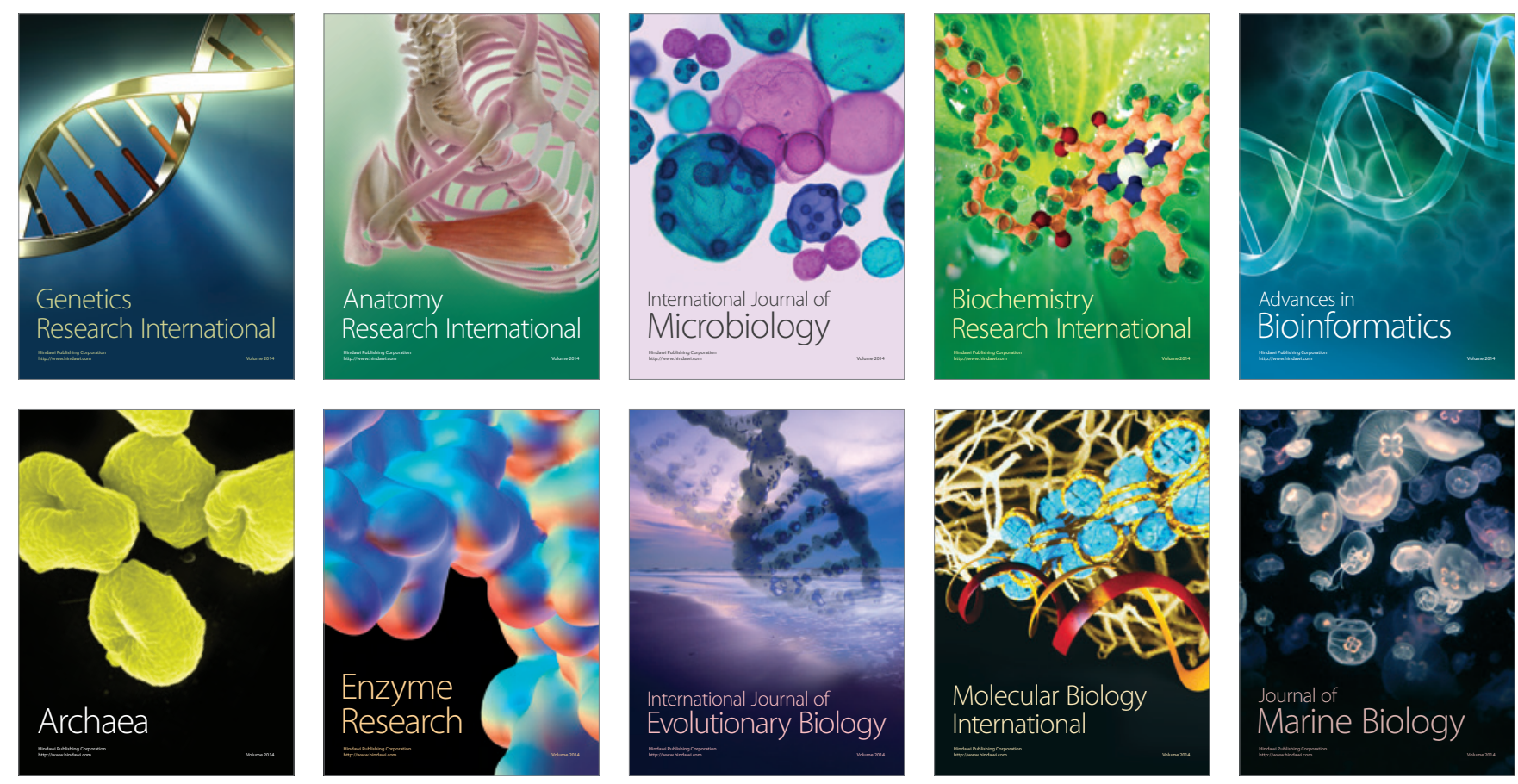